\title{
Malignant sacrococcygeal germ cell tumors in childhood: The Associazione Italiana Ematologia Oncologia Pediatrica (AIEOP) experience
}

\author{
Paolo D'Angelo ${ }^{1}$ () | Maria Debora De Pasquale ${ }^{2}$ () | Francesco Barretta ${ }^{3}$ (i) \\ Maria Carmen Affinita ${ }^{4}$ ( ) | Massimo Conte ${ }^{5}$ ( ) | Patrizia Dall'Igna ${ }^{6}$ () | Andrea Di

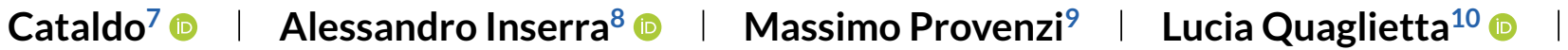 \\ Giovanna Riccipetitoni $^{11}$ | Filippo Spreafico ${ }^{12}$ (1) | Angela Trizzino ${ }^{1}$ | \\ Monica Terenziani ${ }^{12}$ ( ) $\quad$ for the AIEOP MGCT Working Group \\ ${ }^{1}$ Pediatric Hematology and Oncology Unit, ARNAS "Civico, Di Cristina and Benfratelli" Hospitals, Palermo, Italy \\ 2 Pediatric Hematology and Oncology Department, Ospedale Pediatrico Bambino Gesù IRCCS, Rome, Italy \\ 3 Unit of Clinical Epidemiology and Trial Organization, Fondazione IRCCS Istituto Nazionale dei Tumori di Milan, Milan, Italy \\ ${ }^{4}$ Hematology/Oncology Division, Department of Women's and Children's Health, University of Padua, Padua, Italy \\ ${ }^{5}$ Department of Hematology and Oncology, Istituto G. Gaslini, Genoa, Italy \\ ${ }^{6}$ Pediatric Surgery Division, Department of Women's and Children's Health, University of Padua, Padua, Italy \\ ${ }^{7}$ Pediatric Hematology and Oncology Unit, Catania University, Catania, Italy \\ 8 Pediatric Surgery Unit, Ospedale Pediatrico Bambino Gesù, Rome, Italy \\ ${ }^{9}$ Pediatric Hematology and Oncology Unit, Papa Giovanni XXIII Hospital, Bergamo, Italy \\ 10 Pediatric Oncology Unit, Santobono-Pausilipon Hospitals, Naples, Italy \\ ${ }^{11}$ Department of Pediatric Surgery, "Buzzi” Children Hospital, Milan, Italy \\ 12 Pediatric Oncology Unit, Fondazione IRCCS Istituto Nazionale Tumori, Milan, Italy
}

\section{Correspondence}

Paolo D'Angelo, Unità Operativa di Oncoematologia Pediatrica, A.R.N.A.S. Civico, Di Cristina e Benfratelli, Piazza Nicola Leotta, 4, 90127

Palermo, Italy.

Email:oncoematoped@arnascivico.it

\begin{abstract}
Purpose: To evaluate clinical features at diagnosis, prognostic factors, and outcomes of malignant sacrococcygeal germ cell tumors (SC-GCTs) in patients enrolled in the Associazione Italiana Ematologia Oncologia Pediatrica (AIEOP) TCG 2004 protocol.

Patients and Methods: A prospective analysis was conducted on all consecutive patients diagnosed with malignant SC-GCTs between January 2004 and May 2017. Patients with stage I underwent surgery and subsequent surveillance, the others received pediatric cisplatinum-etoposide-bleomycin ( $p$ PEB) regimen and eventual deferred surgery.

Results: Of 45 patients, 35 were females. Age at diagnosis ranged from 1 day to 3.6 years (median 1.6 years); 26 were stage IV. Of 38 patients who underwent surgery, pathology revealed yolk sac tumor (YST) in 27 and teratoma + YST/embryonal carcinoma in 11, while seven patients were diagnosed based on imaging and elevated levels
\end{abstract}

Abbreviations: AFP, alpha-fetoprotein; AIEOP, Associazione Italiana di Ematologia e Oncologia Pediatrica; Cl, confidence interval; COG, Children's Oncology Group; CR, complete remission; EC, embryonal carcinoma; EFS, event-free survival; GCTs, germ cell tumors; IT, immature teratoma; LDH, lactate dehydrogenase; MT, mature teratoma; OS, overall survival; PD, progressive disease; pPEB, pediatric cisplatinum-etoposide-bleomycin; PR, partial remission; SC-GCTs, sacrococcygeal germ cell tumors; YST, yolk sac tumor; $\beta$-hCG, beta-human chorionic gonadotropin 
of alpha-fetoprotein (AFP). Of six patients approached with surgery, only one relapsed and was rescued with first-line chemotherapy. Overall, 38 out of 45 achieved complete remission, three a partial remission, and four were resistant. Ten out of 41 patients who entered remission later relapsed and nine were rescued with a second-line treatment. We observed a global failure percentage of $31 \%$ and a 5 -year overall survival (OS) and event-free survival (EFS) of $95 \%$ and $69 \%$, respectively.

Conclusions: Chemotherapyis generally effective in malignant SC-GCTs, even though almost one-third of our patients experienced events salvageable with second-line treatment. Most of the relapses occurred within 1 year from diagnosis. A close follow up with serial AFP level monitoring should be done for at least 2 years after diagnosis.

KEYWORDS

childhood, malignant neoplasms, prognostic factors, sacrococcygeal germ cell tumors

\section{1 | INTRODUCTION}

Pediatric germ cell tumors (GCTs) are rare neoplasms derived from primordial germ cells, and may arise from the gonads or extragonadal organs. They account for about $3 \%$ of all childhood malignancies, and typically have a bimodal age distribution, with a peak before 3 years of age and a second peak during adolescence. ${ }^{1,2}$ Extragonadal tumors are typically distributed along the midline, suggesting that these tumors arise from the abnormal migration of primordial germ cells from the allantois to the genital crest. The most common site of extragonadal GCTs in childhood is the sacrococcygeal region, followed by the anterior mediastinum, intracranial region, retroperitoneum, neck, and vagina-uterus. ${ }^{2}$ The most common histology of sacrococcygeal GCTs (SC-GCTs) is teratoma, which generally are benign; the most common malignant histology is yolk sac tumor (YST) often admixed with teratoma. ${ }^{1-3}$ The introduction of platinum-based chemotherapy dramatically improved the prognosis for these patients. ${ }^{4-7} \mathrm{We}$ present clinical features at diagnosis, prognostic factors, and outcomes of malignant SC-GCTs enrolled in the second Italian protocol Associazione Italiana Ematologia Oncologia Pediatrica (AIEOP) TCG 2004.

\section{2 | MATERIALS AND METHODS}

The TCG 2004 is a prospective trial conducted at 17 AIEOP centers from January 2004 to May 2017. AIEOP is a cooperative network that brings together all pediatric hematology and oncology centers in Italy. Only 13 centers enrolled cases of malignant SC-GCTs and four of these registered only one patient. The eligibility criteria were: age less than 18 years, informed consent, histological diagnosis of extracranial malignant GCT, histological diagnosis of teratoma (mature and/or immature), but with pathologic values of alpha-fetoprotein (AFP) and/or betahuman chorionic gonadotropin ( $\beta$-hCG), confirming the presence of malignant tissue foci and/or with metastatic localizations and/or with carcinomatous or sarcomatous malignant tissue component histologically proven and with pathologic values of AFP and/or $\beta$-hCG.

All patients diagnosed with malignant SC-GCTs enrolled in the AIEOP TCG 2004 protocol were included in this study. The protocol was approved by the local ethical committees, and informed consent according to institutional guidelines was obtained for all patients and their guardian(s). Demographic and the clinical-pathological characteristics of patients, including sex, age, tumor size $(<9 \mathrm{~cm},>9 \mathrm{~cm})$ at diagnosis, staging, ${ }^{8}$ the site of metastasis, the time of surgery (deferred, not deferred), histology, serum levels of AFP, $\beta$-hCG, and lactate dehydrogenase (LDH), distinguishing between normal and pathologic, and treatment data were collected.

\subsection{Evaluation at diagnosis}

Initial medical evaluation included medical history, physical examination, complete blood count with differential, urinalysis, electrolytes, creatinine, bilirubin, AST, ALT, alkaline phosphatase, total protein, albumin, and measurement of serum levels of LDH, AFP, and $\beta$-hCG. Diagnostic imaging evaluation included ultrasonographic scan (US), and computed tomography (CT) or magnetic resonance imaging (MRI), according to local availability. Initial disease extension was assessed in all patients by US and CT or MRI scan of the pelvis and SC region, a bone scan (omitted in the case of newborns and in all cases where other instrumental investigations indicated there were no injuries to the skeleton), and a chest CT to detect bone and lung metastases. Brain CT scans or MRIs were performed in the presence of neurological symptoms.

\section{2 $\quad$ Treatment and follow up}

Upfront surgery was considered (biopsy in the case of a very large nonsecreting tumor, excision in the case of a tumor considered as 
completely resectable without mutilating surgery), or after chemotherapy in order to remove all residual tumor, preferably after normalization of serum markers. Surgical recommendations included complete excision of the tumor without rupture and/or spillage of tumor cells on the tumor bed, and coccygeal excision, which is considered worldwide to be a key step of the surgical protocol to avoid recurrence. As staging system, the Children's Oncology Group (COG) classification was adopted. ${ }^{8}$ Centralized pathology review was suggested, but was not mandatory.

Subsequent treatments depended on the disease stage: patients with stage I disease and with tumor markers returning to normal level underwent surveillance alone. The others were treated with a pediatric cisplatinum-etoposide-bleomycin (pPEB) regimen, consisting of cisplatin $25 \mathrm{mg} / \mathrm{m}^{2}$ daily on days $1-4$, etoposide $100 \mathrm{mg} / \mathrm{m}^{2}$ daily on days $1-4$, and bleomycin $15 \mathrm{mg} / \mathrm{m}^{2}$ on day 2 , once per cycle. ${ }^{9}$

\section{3 | Response evaluation}

The measurement of serum tumor markers and LDH was recommended after every course, and US or CT/MRI were performed after the second course. CT or MRI scans of the primary site, chest CT scans, and bone scans were repeated after the fourth course, at the suspension of treatment, according to the diagnosis. Response criteria were as reported elsewhere. ${ }^{9}$ The normalization of serum markers and the disappearance of tumor lesions on CT or MRI were classified as a complete remission (CR). Patients with negative imaging and lower tumor markers, or with imaging abnormalities at the primary tumor/regional sites and declining/normalizing tumor markers, were classified as partial remissions (PR). Patients with a greater than $25 \%$ increase in tumor size, new lesions, and rising tumor markers were classified as progressive disease (PD). Patients with PR and residual tumor underwent surgical exploration and subsequent postoperative assessments, and therapy depended on histological findings, which were classified as fibronecrotic tissue, residual teratoma, and viable malignant cells.

\subsection{Subsequent follow up}

Patients were followed up with abdominal ultrasound and chest $X$-rays, clinical examinations, and AFP and $\beta$-hCG assay every 2 months in the first year after completing any treatment, every 3 months in the second year, then 6 monthly until the fifth year. After the fifth year, follow up was at the discretion of the local center.

\subsection{Statistical analysis}

The principal end-points of the study were overall survival (OS) and event-free survival (EFS) probabilities. OS was defined as the time elapsing from diagnosis to death from any cause. Time was censored at the last follow up for patients still alive. EFS was defined as the time elapsing from diagnosis to any event (refractory disease, recurrence, growing teratoma syndrome, or death, whichever occurred first). Time was censored at the last follow up for patients still alive and eventfree. The OS and EFS curves were estimated using the Kaplan-Meier method. Univariable Cox models were performed to assess the association between the EFS and the principal "a priori" selected prognostic variables including sex, age (modeled as restricted cubic spline to obtain flexible fit), ${ }^{10}$ stage (IV vs II-III), bone metastases with or without other metastasis (yes, no), histology (YST only, YST + other), completeness of surgery (excision, biopsy, not done), tumor size $(<9 \mathrm{~cm}$, $\geq 9 \mathrm{~cm}$ ), serum levels of AFP at diagnosis (pathological vs normal), and deferred surgery (yes, no). The median follow up was estimated with the reverse Kaplan-Meier method on OS data.

\section{3 | RESULTS}

A total of 45 patients with SC-GCTs were included in this study. In Table 1, we present the demographic characteristics of the study population. Females were predominant (78\%). The median follow up was 7.4 years (interquartile range 5.1-11.3 years). Age at diagnosis ranged from 1 day to 3.6 years (median 1.6 years); most patients presented advanced stages with $24 \%$ in stage III and $58 \%$ in stage IV, and the lung was by far the most affected distant organ. Thirty-eight underwent surgery at diagnosis: biopsy in 26 and tumor resection (complete in two) in 12 children. For seven patients, diagnosis was based on imaging and the elevated serum level of AFP. Pathology revealed pure YST in 27, mature teratoma (MT) + multiple foci of YST in five, immature teratoma (IT) grade 2 or 3 in five (with multiple foci of YST in four and microfoci in one), and mixed tumor, YST/MT (10\%)/embryonal carcinoma (EC) in one patient only, aged 1.6 years. Thirteen patients showed neurological signs or symptoms at the beginning, all with loss of sphincteric control, linked with paraparesis in three of them. Elevated serum levels of AFP and $\beta$-hCG were found in 40 and five children (all newborns), respectively: this last finding may be due to the physiological $\beta$-hCG maternal circulation, since it was always linked to the neonatal age, and the only patient with an EC component showed normal values. $\mathrm{LDH}$ serum levels resulted pathologic in 33 , normal in nine, and were not evaluated in three (Table 2). Overall, seven patients had an age of less than 3 months at diagnosis. Six of them (five newborns and one 40-day-old girl with multiple malformations), of which two were stage I and four were stage II or III, were approached with surgery only, and these cases are summarized in Table 3. Despite the incompleteness of the resection in four of them, only one relapsed (patient 25). She underwent excision of a large primary tumor mass $(15 \times 12 \times 10 \mathrm{~cm})$ on her second day of life, with minor tumor rupture and bleeding, and grade 3 IT/YST histology was identified; she relapsed locally (with a rise in AFP serum levels) 14 months after surgery, and was rescued with first-line chemotherapy (four courses of pPEB) and subsequent complete resection. Only one newborn of the series underwent chemotherapy after a microscopically incomplete surgery in two steps. The first surgical procedure of incomplete excision of the mass en bloc with coccygectomy was conducted when she was 2 days old; histology revealed a mixed tumor composed of MT and $30 \%$ of YST; the second excision was 
TAB LE 1 Demographic and clinical-pathological characteristics

\begin{tabular}{|c|c|}
\hline & $N=45$ \\
\hline \multicolumn{2}{|l|}{ Sex, n (\%) } \\
\hline Female & $35(78)$ \\
\hline Male & $10(22)$ \\
\hline \multicolumn{2}{|l|}{ Age at diagnosis, years } \\
\hline Median (IQR) & $1.6(1.0-2.0)$ \\
\hline \multicolumn{2}{|l|}{ Neurological signs or symptoms at diagnosis, $\mathrm{n}(\%)$} \\
\hline Loss of the sphincterial control & $10(22)$ \\
\hline Loss of the sphincterial control and paraparesis & $3(7)$ \\
\hline None & $32(71)$ \\
\hline \multicolumn{2}{|l|}{ AFP serum level at Dx, $n(\%)$} \\
\hline High & $40(89)$ \\
\hline Normal & $5(11)$ \\
\hline
\end{tabular}

\section{$\beta$-hCG serum level at Dx, $\mathrm{n}(\%)$}

High

Normal

Not done

LDH serum level at Dx, $\mathrm{n}(\%)$

High

Normal

Not done

$3(7)$

Tumor size at $\mathrm{Dx}$ ( $\max$ diameter), $\mathrm{n}(\%)$

$<9 \mathrm{~cm}$

$\geq 9 \mathrm{~cm}$

NA

\section{Staging, $\mathrm{n}(\%)$}

I

II

III

IV

Site of metastases, $\mathrm{n}(\%)^{\mathrm{a}}$

Lung

Liver

Bone

\section{Timing of surgery, $\mathbf{n}(\%)$}

Deferred

Not deferred

\section{Completeness of surgery, $\mathrm{n}(\%)$}

Complete excision

Incomplete excision

Biopsy only

Not done

(Continues)
TABLE 1 (Continued)

\begin{tabular}{ll} 
Histology, $\mathrm{n}(\%)^{\mathrm{b}}$ & $\mathrm{N}=45$ \\
YST only & $27(71)$ \\
YST + other & $11(29)$ \\
\hline
\end{tabular}

Abbreviations: AFP, alpha-fetoprotein; Dx, diagnosis; EC, embryonal carcinoma.; IQR, interquartile range; $L D H$, lactate dehydrogenase; NA, not available; YST, yolk sac tumor; $\beta$-hCG, beta-human chorionic gonadotropin.

${ }^{a}$ Each percentage was calculated on the whole population of patients (many of them showed combined metastases).

${ }^{\text {b }}$ On 38 available patient data only.

performed to remove the pelvic residual mass when she was 2 months old, and chemotherapy with four courses of pPEB then completed her treatment. All seven patients aged $<3$ months at diagnosis are in $C R$, six in first and one in second continuous $C R$.

Overall, 39 out of 45 (87\%) patients underwent chemotherapy and four suffered from disease progression. A good response to chemotherapy was achieved in 35 patients (CR or PR), 31 of which underwent deferred surgery. Globally, thirty-seven patients entered CR (two of them with surgery only), four obtained PR (and achieved $C R$ through deferred surgery), and four were nonresponders. Of these four nonresponders, the first one aged 2 years, YST, stage III for lymph nodes and peritoneal involvement, showed local progression, an increase in AFP levels, and multiple central nervous system metastases; a second-line treatment according to the ICE regimen ${ }^{11}$ proved ineffective and she died of PD 6 months after the diagnosis. The second one, aged 2.7 years at diagnosis, YST, stage IV for bilateral lung metastases, which showed local and marker-related progression after three courses of pPEB, received a second-line therapy and obtained a second continuous $\mathrm{CR}$; unfortunately she died due to a fatal ventricular arrhythmia, following a heart transplant, performed because of heart failure, more than 10 years after the discontinuation of antiblastic treatment. The third nonresponder patient, YST, stage IV for liver metastases, showed local progression and an increase in serum marker levels. She underwent deferred surgery (resection of the primary en bloc with coccigectomy) and shifted to second-line chemotherapy according to the ICE schedule, which enabled a second continuous CR. The fourth patient, YST, stage IV for liver, bone, and lung metastases, showed a mixed response of the primary and metastatic disease and an increase of AFP serum levels after four courses, and was rescued with two courses of ICE, surgery, and subsequent high-dose chemotherapy (HDCT) and autologous peripheral blood stem cells transplant (PBSCT).

Of 40 patients with abnormal AFP at diagnosis, 35 showed a complete normalization, two a partial reduction, and three an increase of serum level.

Overall, of 41 patients entering a remission status (six infants with surgery only), 10 (24\%) relapsed: nine with localized recurrence (one combined with bone metastases) and one with exclusively metastatic spread, and all of them showed increasing serum levels of AFP (Table 4). One was the already mentioned newborn that, following the local relapse and marker increase ( 14 months after surgery), received four 
TAB LE 2 Marker levels measured in the whole series

\begin{tabular}{|c|c|c|c|c|c|c|}
\hline $\begin{array}{l}\text { Patient } \\
\text { number }\end{array}$ & $\begin{array}{l}\text { Age at } \\
\text { Diagnosis } \\
\text { (years) }\end{array}$ & $\begin{array}{l}\text { AFP serum level } \\
\text { presurgery } \\
(\mathrm{ng} / \mathrm{mL})\end{array}$ & Surgery & $\begin{array}{l}\text { AFP serum level } \\
\text { postsurgery } \\
(\mathrm{ng} / \mathrm{mL})\end{array}$ & $\begin{array}{l}\text { Beta-hCG serum } \\
\text { level at diagnosis } \\
\text { (IU/mL) }\end{array}$ & $\begin{array}{l}\text { LDH serum leve } \\
\text { at diagnosis } \\
\text { (IU/L) }\end{array}$ \\
\hline 1 & 2.0 & 80514 & Biopsy & NA & Normal & 1241 \\
\hline 2 & 0.9 & 39997 & Biopsy & NA & Normal & 1164 \\
\hline 3 & 1.4 & 106877 & Biopsy & NA & Normal & 1363 \\
\hline 6 & 2.5 & 28723 & Biopsy & NA & Normal & Normal \\
\hline 7 & 2.0 & $>50000$ & No & NA & Normal & 1062 \\
\hline 8 & 1.4 & 133770 & Incomplete excision & 325 & Normal & 730 \\
\hline 9 & 1.8 & 141847 & Biopsy & NA & Normal & 704 \\
\hline 13 & 1.5 & 262140 & No & NA & Normal & Normal \\
\hline 14 & 1.2 & 114619 & Biopsy & NA & Normal & Normal \\
\hline 15 & 2.2 & 61670 & No & NA & Normal & Normal \\
\hline 16 & 1.7 & 138951 & No & NA & Normal & 699 \\
\hline 17 & 1.7 & $>150000$ & Biopsy & NA & $\mathrm{Nd}$ & $\mathrm{Nd}$ \\
\hline 18 & 0.9 & 20239 & Biopsy & NA & Normal & 1435 \\
\hline 19 & 1.6 & 35160 & Biopsy & NA & Normal & 940 \\
\hline 20 & 0.0 & $75700^{a}$ & Incomplete excision & 7447 & 23 & 872 \\
\hline 27 & 1.1 & 187890 & Biopsy & NA & Normal & 689 \\
\hline 28 & 1.5 & 3754 & Incomplete excision & 283 & Normal & 1003 \\
\hline 29 & 0.1 & $563^{a}$ & Complete excision & 115 & Normal & 900 \\
\hline 30 & 3.3 & 24000 & Biopsy & NA & Normal & 900 \\
\hline 31 & 0.0 & $6936^{a}$ & Complete excision & 937 & Normal & 1436 \\
\hline 32 & 1.0 & 204131 & Biopsy & NA & Normal & 1066 \\
\hline 33 & 0.7 & 37323 & Incomplete excision & 2200 & $\mathrm{Nd}$ & $\mathrm{Nd}$ \\
\hline 34 & 3.6 & 17208 & Biopsy & NA & Normal & Normal \\
\hline 35 & 1.5 & 167400 & Biopsy & NA & Normal & Normal \\
\hline 36 & 1.8 & 53950 & Biopsy & NA & Normal & 705 \\
\hline 37 & 2.3 & 60500 & No & NA & Normal & 691 \\
\hline 38 & 0.0 & 201800 & Incomplete excision & 71380 & 81 & $\mathrm{Nd}$ \\
\hline 39 & 1.6 & 60500 & No & NA & Normal & 914 \\
\hline 40 & 0.0 & $31244^{a}$ & Incomplete excision & 1351 & 38 & 430 \\
\hline 41 & 3.3 & 25086 & Biopsy & NA & Normal & 496 \\
\hline 42 & 1.3 & 235180 & Biopsy & NA & Normal & 952 \\
\hline
\end{tabular}


TABLE 2 (Continued)

\begin{tabular}{|c|c|c|c|c|c|c|}
\hline $\begin{array}{l}\text { Patient } \\
\text { number }\end{array}$ & $\begin{array}{l}\text { Age at } \\
\text { Diagnosis } \\
\text { (years) }\end{array}$ & $\begin{array}{l}\text { AFP serum level } \\
\text { presurgery } \\
\text { (ng/mL) }\end{array}$ & Surgery & $\begin{array}{l}\text { AFP serum level } \\
\text { postsurgery } \\
\text { (ng/mL) }\end{array}$ & $\begin{array}{l}\text { Beta-hCG serum } \\
\text { level at diagnosis } \\
\text { (IU/mL) }\end{array}$ & $\begin{array}{l}\text { LDH serum level } \\
\text { at diagnosis } \\
\text { (IU/L) }\end{array}$ \\
\hline 43 & 2.6 & 55619 & Biopsy & NA & Normal & Normal \\
\hline 44 & 1.9 & 15737 & Incomplete excision & 4437 & Normal & 575 \\
\hline 45 & 2.4 & 9775 & Biopsy & NA & Normal & 731 \\
\hline
\end{tabular}

Abbreviations: AFP, alpha-fetoprotein; hCG, human chorionic gonadotropin; LDH, lactate dehydrogenase; NA, not applicable; Nd, not done.

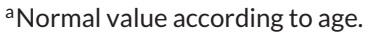

TAB LE 3 Characteristics of patients treated with surgery only

\begin{tabular}{|c|c|c|c|c|c|c|c|}
\hline Pt no. & $\begin{array}{l}\text { Age at } \\
\text { Dx }\end{array}$ & Initial surgery & Stage & $\begin{array}{l}\text { AFP } \\
\text { (ng/mL) }\end{array}$ & Histology at Dx & Relapse & $\begin{array}{l}\text { Subsequent } \\
\text { therapy }\end{array}$ \\
\hline 20 & 1 day & Incompl for micro residue & II & 75700 & G3 IT with multiple foci of YST & No & \\
\hline 26 & 3 days & Incompl for marginal surg & II & 154598 & G2 IT with multiple foci of YST & No & \\
\hline 29 & 40 days & Complete surg & I & 563 & G2 IT with multiple foci of YST & No & \\
\hline 38 & 3 days & Incompl for macro residue & III & 201800 & G3 IT with microfoci of YST & No & \\
\hline
\end{tabular}

Abbreviations: AFP, alpha-fetoprotein; Dx, diagnosis; G, grading; Incompl, incomplete; IT, immature teratoma; macro, macroscopic; micro, microscopic; mo, months; MT, mature teratoma; pPEB, pediatric cisplatinum-etoposide-bleomycin; Pt, patient; surg, surgery; YST, yolk sac tumor.

courses of pPEB and subsequent complete surgery; nine were rescued with a second-line treatment according to the ICE regimen, plus surgery in six of them, and HDCT with subsequent autologous PBSCT in six. Three children also underwent local radiotherapy (see Table 3). ${ }^{11}$
Only one of the relapsed patients (aged 1.7 years at diagnosis, YST, stage IV for bilateral lung metastases, AFP > $150000 \mathrm{ng} / \mathrm{mL}$ at diagnosis) died due to progression of the disease after several more lines of treatment. Of the remaining nine patients, eight are in second contin-

TAB LE 4 Characteristics of the relapsed patients

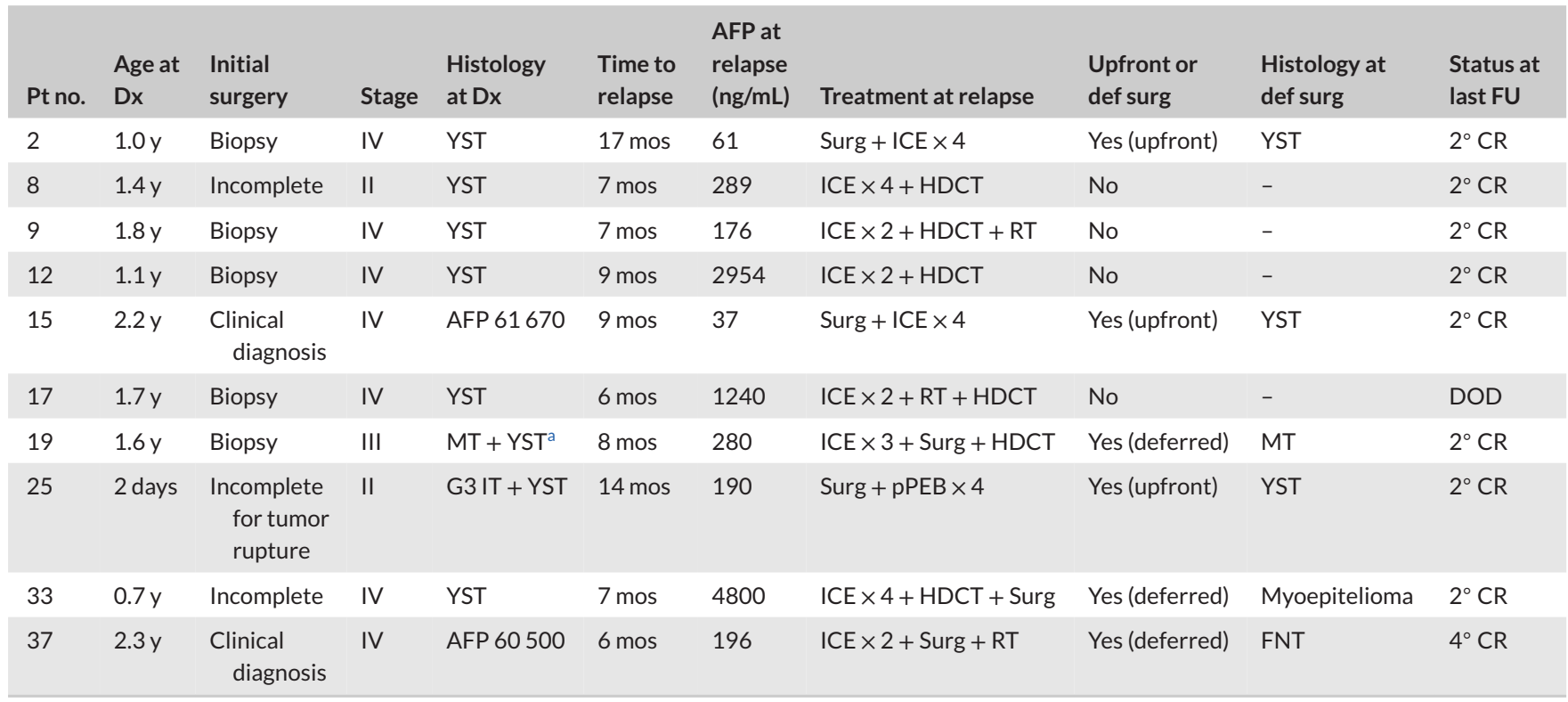

Abbreviations: AFP, alpha-fetoprotein (ng/mL); CR, complete remission; def, deferred; DOD, dead of disease; Dx, diagnosis; FNT, fibronecrotic tissue; G3, grading 3; HDCT, high-dose chemotherapy; ICE, ifosfamide-carboplatinum-etoposide; IT, immature teratoma; mos, months; MT, mature teratoma; pPEB, pediatric cisplatinum-etoposide-bleomycin; Pt, patient; RT, radiotherapy; Surg, surgery; y, years; YST, yolk sac tumor.

${ }^{a} \mathrm{AFP}$ at diagnosis $35160 \mathrm{ng} / \mathrm{mL}$. 
TAB LE 5 Characteristics of the patients with associated neurological involvement at diagnosis

\begin{tabular}{|c|c|c|c|c|c|c|}
\hline Pt no. & $\begin{array}{l}\text { Age at } \\
\text { Dx }\end{array}$ & $\begin{array}{l}\text { Neurological signs and } \\
\text { symptoms at Dx }\end{array}$ & Stage & Sites of MTS & Initial surgical approach & $\begin{array}{l}\text { Neurological outcome at } \\
\text { follow up }\end{array}$ \\
\hline 3 & $1.4 \mathrm{y}$ & Sphincterial disorders & IV & Lung + bone & Biopsy & Complete recovery \\
\hline 10 & $2.0 y$ & $\begin{array}{l}\text { Paraparesis and } \\
\text { urination disorders }\end{array}$ & III & & Biopsy + laminectomy & Persistence of disturbances \\
\hline 12 & $1.0 \mathrm{y}$ & $\begin{array}{l}\text { Paraparesis and } \\
\text { sphincterial disorders }\end{array}$ & IV & $\begin{array}{l}\text { Lung + bone + } \\
\text { liver }\end{array}$ & Biopsy & Persistence of disturbances \\
\hline 13 & $1.5 \mathrm{y}$ & $\begin{array}{l}\text { Paraparesis and } \\
\text { sphincterial disorders }\end{array}$ & III & & None & Persistence of disturbances \\
\hline 21 & $1.3 y$ & Urinary incontinence & IV & Lung + bone & Biopsy & Persistence of disturbances \\
\hline 40 & 2 days & $\begin{array}{l}\text { Equin foot and } \\
\text { neurological bladder }\end{array}$ & II & & $\begin{array}{l}\text { Two steps resec with } \\
\text { microsc residue }\end{array}$ & Persistence of disturbance \\
\hline 42 & $1.3 y$ & $\begin{array}{l}\text { Severe sphincterial } \\
\text { disorders }\end{array}$ & IV & Lung + bone & Biopsy & Complete recovery \\
\hline 43 & $2.6 y$ & Sphincterial disorders & IV & Lung & Biopsy & Complete recovery \\
\hline 45 & $2.4 y$ & Sphincterial disorders & IV & Lung & Biopsy & Complete recovery \\
\hline
\end{tabular}

Abbreviations: Dx, diagnosis; microsc, microscopic; MTS, metastases; Pt, patient; resec, resection; y, years.

uous $\mathrm{CR}$ and one is in fourth continuous $\mathrm{CR}$ after two further relapses and related systemic and local treatments. Concerning the patient 33 showed a new increase of serum AFP 2 months after stop therapy and a relapse in the soft tissues of the left buttock was diagnosed. She underwent chemotherapy with four courses of ICE and subsequent HDCT and PBSCT, with normalization of AFP. A subsequent surgical look on the left buttock revealed a residual neoplastic proliferation characterized by negativity of markers for endodermic sinus tumor (AFP, SALL4, glypican) and more generally for tumors of the germinal line (ОСТ3/4 and CD30), while positivity of cytokeratins, EMA, smooth muscle actin, and focally protein $\mathrm{S} 100$ was suggestive of a nonmalignant neoplastic differentiation, called "myoepithelioma." No treatment has been done for this completely resected benign tumor.

Five out of 13 patients with neurological signs and symptoms at diagnosis (loss of sphincteric control with paraparesis in three cases) recovered completely (Table 5).

Five-year OS and EFS were 95\% (95\% confidence interval [CI]: 89$100 \%$ ) and $69 \%$ (95\% Cl: 56-84\%), respectively (Figure 1). None of the prognostic factors that we took into consideration showed a statistically significant impact on prognosis in univariable analysis (Table 6).

\section{4 | DISCUSSION}

Sacrococcygeal teratomas are the most common GCT of infancy and early childhood, accounting for $40 \%$ of all non-CNS GCTs. While most of these tumors have only mature or IT elements, $11-35 \%$ could contain malignant elements, with this proportion increasing with age. The most commonly found malignant element is YST. Rarely, these tumors may include another malignant component, usually EC or even more rarely a nongerm cell/somatic malignant component such as primitive neuroectodermal tumor. The earlier published series on GCTs displayed very poor results in children with malignant SC-GCTs, ${ }^{7,13,14}$ yet more recently this site is no longer regarded as a poor prognostic factor of survival after the introduction of platinum-based chemotherapy regimens. ${ }^{14,15}$ These results were achieved by the administration of chemotherapy in almost all patients, when the size and site of the primary tumor and the high rate of regional lymph node involvement or concomitant distant metastases generally preclude a complete upfront surgical resection. The impact of clinically advanced disease at diagnosis is well known in this site: the incidence of metastasis at diagnosis in our series (58\%) is higher than that reported in the German MAKEI protocol (where it is $45 \%$ ), ${ }^{16}$ but fairly similar to the $59 \%$ level reported in the North American COG series. ${ }^{17,18}$

The introduction of multidrug chemotherapy has dramatically improved the outcome of pediatric patients with malignant GCTs. ${ }^{3,6,7}$ The 5 -year survival for these patients now approaches $90 \%$, and the identification of patients at a high risk of treatment failure is a crucial challenge.

Several reports have aimed at defining prognostic factors in malignant GCTs. ${ }^{4,5,13,14}$ High levels of serum AFP (>10 $000 \mathrm{ng} / \mathrm{mL}$ ), extragonadal site, the stage of disease, the size of the primary tumor, and the 


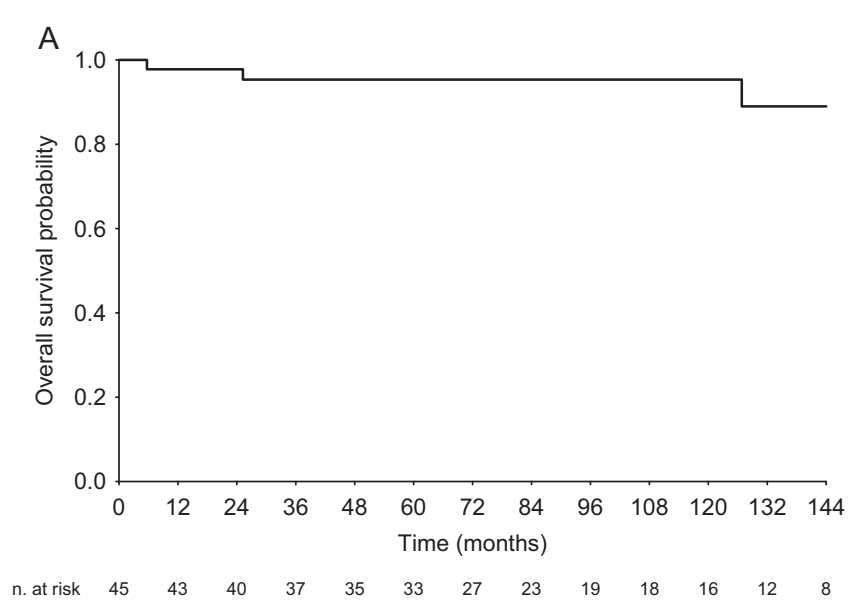

TAB LE 6 Results of the univariable Cox models for event-free survival

\begin{tabular}{|c|c|c|c|}
\hline & HR & $95 \% \mathrm{Cl}$ & $P$ \\
\hline Sex & & & .366 \\
\hline Male vs female & 0.50 & $0.11-2.24$ & \\
\hline Age at diagnosis (years) & & & .697 \\
\hline 2 vs $1^{a}$ & 0.92 & $0.51-1.97$ & \\
\hline Stage & & & .359 \\
\hline IV vs II-III & 1.72 & $0.54-5.49$ & \\
\hline Bone metastasis & & & .274 \\
\hline Yes vs no & 1.91 & $0.60-6.12$ & \\
\hline Histology ${ }^{b}$ & & & .280 \\
\hline YST vs YST + other & 2.31 & $0.51-10.55$ & \\
\hline Surgery & & & .945 \\
\hline Excision vs not done & 1.32 & $0.24-7.19$ & \\
\hline Biopsy vs not done & 1.13 & $0.24-5.31$ & \\
\hline Tumor size $(\mathrm{cm})^{c}$ & & & .959 \\
\hline$\geq 9$ vs $<9$ & 1.03 & $0.31-3.43$ & \\
\hline AFP serum level at diagnosis & & & .606 \\
\hline Pathologic vs normal & 1.71 & $0.22-13.00$ & \\
\hline Deferred surgery & & & .199 \\
\hline Yes vs no & 0.47 & $0.15-1.49$ & \\
\hline
\end{tabular}

Abbreviations: AFP, alpha-fetoprotein; $\mathrm{Cl}$, confidence interval; $\mathrm{HR}$, hazard ratio; $P$, Wald test $P$-value; UK, unknown; $Y S T$, yolk sac tumor.

aModeled as restricted cubic spline; values reported third versus first quartile.

FIGURE 1 Overall survival (panel A) and event-free survival (panel B) curves for the whole series

${ }^{\mathrm{b}}$ On 38 patients with histological diagnosis.

${ }^{\mathrm{C}}$ One patient excluded due to missing data.

completeness of surgery are generally considered important prognostic factors. ${ }^{19,20}$

In our series, it is noteworthy that only one patient out of six approached only with surgical treatment (due to the young age) relapsed, despite the incompleteness of the resection and stage disease $>\mathrm{I}$ in most of them, in contrast with the observation of Egler et $\mathrm{al}^{20}$ of relapse in all the patients other than stage I treated with surgery only. This small subgroup of patients, which has not received any kind of adjuvant treatment precisely because of their rarity and the interest they arouse, should be the subject of an international cooperative study.

The present series of patients registered in the AIEOP TCG 2004 protocol confirms that malignant SC-GCTs have a fairly good overall prognosis with a risk-adapted therapeutic strategy showing a 5-year OS of $95 \%$. We observed an unsatisfactory EFS probability of $69 \%$, even if this was an improvement over the previous Italian cooperative protocol. Particularly, we observed a high proportion of refractory (almost 10\%) and relapsing patients (24\%), which thus defined a global percentage of treatment failure above $30 \%$. This could be due to unfavorable characteristics of our setting of patients at diagnosis, especially the higher percentage of metastatic disease (58\%) with involvement also of bone and liver (respectively, in six and seven patients), and the number of patients with locally advanced disease (stage III was diagnosed in 11 out of 45 patients). Perhaps the reduction of the therapeutic burden, with lower doses of bleomycin and etoposide, might have played a role in determining an unexpected number of events. Probably due to the small sample size and number of events, leading to underpowered tests, we were unable to identify any prognostic factors nor, in particular, the presence of bone involvement.

In light of the above, the main goal we would like to pursue in our next study is improved EFS. It is extremely important to collect and examine more cases treated with surgery alone in order to improve the definition of prognostic factors for this small group of patients. But in order to gather sufficient data, multicenter intergroup collaboration would be essential.

\section{ACKNOWLEDGMENTS}

The authors gratefully acknowledge and thank other colleagues at participating institutions for their help in identifying all of the sacrococcygeal tumors that we report herein. Without their help, we would not have been able to complete this work: Fraia Melchionda (Pediatric Hematology and Oncology, University of Bologna), Martina Di Martino (Pediatric Hematology and Oncology, 2nd University of Naples), 
Patrizia Bertolini (Pediatric Hematology and Oncology, University of Parma), and Eleonora Basso (Pediatric Hematology and Oncology, University of Turin). The authors wish to thank Prof Matthew Furfine for revising the manuscript, and the Parents' Association "A.S.L.T.I. - Liberi di crescere" Onlus for financial support.

\section{AUTHOR CONTRIBUTIONS}

Paolo D’Angelo, Maria Debora De Pasquale, Angela Trizzino, Filippo Spreafico, and Monica Terenziani contributed to conception, data collection and analysis, and writing of the manuscript. Maria Carmen Affinita, Massimo Conte, Patrizia Dall'Igna, Andrea Di Cataldo, Alessandro Inserra, Massimo Provenzi, Lucia Quaglietta, and Giovanna Riccipetitoni contributed to data collection and critical review of the manuscript. Francesco Barretta contributed to statistical analysis and critical review of the manuscript.

\section{DATA AVAILABILITY STATEMENT}

The data that support the findings of this study are available on request from the corresponding author. The data are not available for publication due to privacy or ethical restrictions.

\section{CONFLICT OF INTEREST}

The authors declare that there is no conflict of interest.

\section{ORCID}

Paolo D'Angelo (DD https://orcid.org/0000-0002-4021-8472

Maria Debora De Pasquale (D) https://orcid.org/0000-0003-1082-6810

Francesco Barretta (D) https://orcid.org/0000-0002-7797-161X

Maria Carmen Affinita (D) https://orcid.org/0000-0003-1680-0240

Massimo Conte (D) https://orcid.org/0000-0001-9405-7339

Patrizia Dall'Igna (D) https://orcid.org/0000-0002-3822-3272

Andrea Di Cataldo (D) https://orcid.org/0000-0002-4509-3066

Alessandro Inserra (D) https://orcid.org/0000-0002-5663-8674

Lucia Quaglietta (D) https://orcid.org/0000-0002-4354-8979

Filippo Spreafico (D) https://orcid.org/0000-0002-5587-3509

Monica Terenziani (D) https://orcid.org/0000-0002-7080-6718

\section{REFERENCES}

1. Schneider DT, Terenziani M, Cecchetto G, Olson TA. Gonadal and extragonadal germ cell tumors, sex cord stromal and rare gonadal tumors. In: Schneider DT, Brecht IB, Olson TA, Ferrari A, eds. Rare Tumors in Children and Adolescents. Berlin: Springer-Verlag; 2012:327402.

2. Olson TA, Murray MJ, Rodriguez-Galindo C, et al. Pediatric and adolescent extracranial germ cell tumors: the road to collaboration. J Clin Oncol. 2015;33:3018-3028.

3. Lo Curto M, Lumia F, Alaggio R, et al. Malignant germ cell tumors in childhood: results of the first Italian cooperative study "TCG91". Med Pediatr Oncol. 2003;41:417-425.

4. Frazier AL, Hale JP, Rodriguez-Galindo C, et al. Revised risk classification for pediatric extracranial germ cell tumors based on 25 years of clinical trial data from the United Kingdom and United States. J Clin Oncol. 2015;33:195-201.

5. Calaminus G, Schneider DT, Bokkerink JPM, et al. Prognostic value of tumor size, metastases, extension into bone, and increased tumor marker in children with malignant sacrococcygeal germ cell tumors: a prospective evaluation of 71 patients treated in the German cooperative protocols Maligne Keimzelltumoren (MAKEI) 83/86 and MAKEI 89. J Clin Oncol. 2003;21:781-786.

6. Marina N, Fontanesi J, Kun L, et al. Treatment of childhood germ cell tumors. Review of the St. Jude experience from 1979 to 1988. Cancer. 1992;70:2568-2575.

7. Mann JR, Pearson D, Barrett A, Raafat F, Barnes JM, Wallendszus KR Results of the United Kingdom Children's Cancer Study Group's malignant germ cell tumor studies. Cancer. 1989;63:1657-1667.

8. Frazier AL, Rumcheva P, Olson T, et al. Application of the adult international germ cell classification system to pediatric malignant nonseminomatous germ cell tumors: a report from the Children's Oncology Group. Pediatr Blood Cancer. 2008;50:746-751.

9. Terenziani M, De Pasquale MD, Bisogno G, et al. Malignant testicular germ cell tumors in children and adolescents: the AIEOP (Associazione Italiana Ematologia Oncologia Pediatrica) protocol. Urol Oncol. 2018;36:502-513.

10. Durrleman S, Simon R. Flexible regression models with cubic splines. Stat Med. 1989;8(5):551-561.

11. De Pasquale MD, D'Angelo P, Crocoli A, et al. Salvage treatment for children with relapsed/refractory germ cell tumors: the Associazione Italiana Ematologia Oncologia Pediatrica (AIEOP) experience. Pediatr Blood Cancer. 2019;18:e28125.

12. Blohm ME, Vesterling-Hörner D, Calaminus G, Göbel U. Alpha 1fetoprotein (AFP) reference values in infants up to 2 years of age. Pediatr Hematol Oncol. 1998;15(2):135-142.

13. Baranzelli MC, Flamant F, De Lumley L, Le Gall E, Lejars O. Treatment of nonmetastatic, non-seminomatous malignant germ-cell tumours in childhood: experience of the "Societe Francaise d'Oncologie Pediatrique" MGCT 1985-1989 study. Med Pediatr Oncol. 1993;21:395401.

14. Brodeur GM, Howarth CB, Pratt CB, Caces J, Hustu HO. Malignant germ cell tumors in 57 children and adolescents. Cancer. 1981;48:1890-1898.

15. Gobel U, Schneider DT, Calaminus G, et al. Multimodal treatment of malignant sacrococcygeal germ cell tumors: a prospective analysis of 66 patients of the German cooperative protocols MAKEI 83/86 and 89. J Clin Oncol. 2001;19:1943-1950.

16. Rescorla F, Billmire D, Stolar C, et al. The effect of cisplatin dose and surgical resection in children with malignant germ cell tumors at the sacrococcygeal region: a pediatric intergroup trial (POG 9049/CCG 8882). J Pediatr Surg. 2001;36:12-17.

17. Marina N, London WB, Frazier AL, et al. Prognostic factors in children with extragonadal malignant germ cell tumors: a pediatric intergroup study. J Clin Oncol. 2006;24:2544-2548.

18. De Corti F, Sarnacki S, Patte C, et al. Prognosis of malignant sacrococcygeal germ cell tumours according to their natural history and surgical management. Surg Oncol. 2012;21:31-37.

19. Baranzelli MC, Kramar A, Bouffet E, et al. Prognostic factors in children with localized malignant nonseminomatous germ cell tumors. $J$ Clin Oncol. 1999;17:1212.

20. Egler RA, Gosiengfiao Y, Russell H, Wickiser JE, Frazier AL. Is surgical resection and observation sufficient for stage I and II sacrococcygeal germ cell tumors? A case series and review. Pediatr Blood Cancer 2017;64(5):e26311.

How to cite this article: D'Angelo P, Debora De Pasquale $M$, Barretta F, et al. Malignant sacrococcygeal germ cell tumors in childhood: The Associazione Italiana Ematologia Oncologia Pediatrica (AIEOP) experience. Pediatr Blood Cancer. 2020;e28812. https://doi.org/10.1002/pbc.28812 